rev.relac.int.estrateg.segur.9(2):143-164,2014

\title{
EL PÉNDULO DESCENTRALIZACIÓN-RECENTRALIZACIÓN Y SU APLICACIÓN A LA REFORMA EDUCATIVA EN MÉXICO
}

\author{
Juan C. Olmeda ${ }^{* *}$
}

\section{RESUMEN}

Durante las últimas décadas del siglo XX los países de América Latina experimentaron profundos procesos de descentralización en aspectos administrativos, políticos y fiscales. Más recientemente, sin embargo, los ejecutivos nacionales en varios países de la región han impulsado políticas de recentralización con la intención de recuperar prerrogativas y responsabilidades. El objetivo de este artículo es analizar estas dinámicas de la recentralización, considerando la creciente literatura que en los últimos años ha proliferado para explicar dichos procesos. Con base en dichos trabajos se identifican tres hipótesis que permiten explicar la recentralización. De acuerdo con dichas hipótesis estaría motivada, respectivamente, por existencia de gobierno unificado, por la estabilización de

Este artículo es resultado de una investigación más extensa que el autor actualmente realiza sobre procesos de recentralización en América Latina.

** Profesor-Investigador, Centro de Estudios Internacionales, El Colegio de México. Email: jcruzo@colmex.mx. 
la economía luego de una crisis hiperinflacionaria o por la intención de ejecutivo federal de castigar a gobiernos subnacionales controlados por la oposición. A estas se podría agregar una cuarta, que propone a la recentralización como una respuesta frente a la incapacidad de los gobiernos subnacionales de prestar correctamente los servicios que le fueron transferidos. Con base en este marco se analiza el reciente proceso recentralizador observado actualmente en el sector educativo en México.

Palabras clave: Descentralización, recentralización, México, Peña Nieto, educación

\title{
THE PENDULUM DE-CENTRALIZATION, RE-CENTRALIZATION AND ITS APPLICATION TO THE EDUCATION REFORM IN MEXICO
}

\begin{abstract}
During the last decades of the XX century, Latin American countries experienced significant decentralization processes in terms of administrative, political and fiscal affairs. More recently, however, national executives in many countries in the region encouraged recentralization policies with the goal of regaining prerrogatives and responsibilities. The goal of this article is to study this recentralization trend, building on the literature that during the last years has attempted to explain these recentralization dynamics. Three hypothesis can be derived from those works in order to identify the reasons behind recentralization. According to these hypothesis, recentralization could be the result of the existence of unified government, the consequence of a stabilized economy after a hyperinflationary crisis or the result of national executive's intention to punish subnational government controlled by the opposition. A fourth additional hypothesis states that recentralization can emerge as a result of the imposibility of subnational governments to deliver those services previously decentralized. Taking into account this conceptual framework the article analizes some recent recentralization measures observed nowadays in Mexico in relation to the education sector.
\end{abstract}

Keywords: Decentralization, recentralization, Mexico, Peña Nieto, education

\section{O PÊNDULO DESCENTRALIZAÇÃO- RECENTRALIZAÇÃO E SUA APLICAÇÃO NA REFORMA EDUCACIONAL NO MÉXICO}

\section{RESUMO}

Durante as últimas décadas do século XX os países da América Latina experimentaram uma profunda descentralização em aspectos administrativos, políticos e fiscais. Mais recentemente, no entanto, os executivos nacionais em vários países da região têm impulsionado políticas de 
recentralização com a intenção de recuperar as prerrogativas e responsabilidades. O objetivo deste trabalho é analisar essas dinâmicas de recentralização, considerando a crescente literatura que nos últimos anos tem proliferado para, assim, explicar esses processos. Com base nestes trabalhos, identificam-se três hipóteses que possibilitam explicar a recentralização. De acordo com estas hipóteses ela estaria motivada, respetivamente, pela existência de um governo unificado, pela estabilidade da economia após uma crise hiperinflacionária ou pela intenção do executivo federal de castigar governos subnacionais controlados pela oposição. Também podese adicionar uma quarta, que propõe a recentralização como uma resposta à incapacidade dos governos subnacionais para fornecer adequadamente os serviços que thes foram transferidos. Com base neste quadro, analisa-se o recente processo recentralizador observado atualmente no setor educacional do México.

Palavras-chave: Descentralização, recentralização, México, Peña Nieto, educação

\section{INTRODUCCIÓN}

Durante las últimas décadas del siglo XX, los países latinoamericanos experimentaron importantes transformaciones políticas y económicas. En muchos casos se dieron transiciones de gobiernos autoritarios a regímenes democráticos. En otros se hizo también visible la adopción de reformas económicas inspiradas en las recetas emanadas del "Consenso de Washington" y orientadas al mercado. En este contexto, casi todos los países citados implementaron políticas de descentralización en los ámbitos administrativo, fiscal y político que redefinieron de manera importante la relación entre niveles de gobierno e hicieron ganar poder a nuevos actores en la política nacional. Dicho proceso ha sido estudiado de manera sustantiva por una amplia literatura que ha florecido desde entonces.

Más recientemente, sin embargo, diferentes autores han comenzado a señalar la puesta en marcha de procesos de recentralización, orientados en la mayoría de los casos a revertir la particular distribución de poder, autonomía y facultades consolidadas en las décadas anteriores. El objetivo del presente artículo es realizar un análisis de estos emergentes procesos de recentralización en la región y considerar las razones a partir de las cuales se explican. Asimismo, se propone aplicar algunas de las nociones presentadas en la creciente literatura acerca de la recentralización para analizar una serie de dinámicas que están teniendo lugar actualmente en México, en particular en el ámbito de la provisión de educación. Argumentamos que, a diferencia de lo propuesto en la literatura sobre la temática, para el caso mexicano una de las hipótesis que mejor parece explicar los intentos recentralizadores es la incapacidad de los estados (desde la perspectiva del centro) de llevar a cabo de manera adecuada la provisión de los servicios públicos que les fueron transferidos en décadas anteriores. Finalmente, proponemos algunas conclusiones y posibles escenarios pensando en el futuro próximo. 


\section{CONCEPTUALIZANDO LA RECENTRALIZACIÓN}

Como ya ha sido señalado, en el período entre 1980 y 2000 los procesos de descentralización dominaron en la agenda de reformas de los países de América Latina, en un contexto en el que dichas políticas eran incentivadas por organismos internacionales y se estaban desarrollando también en otras partes del planeta. En el caso de la región latinoamericana, dicha descentralización coincidió en muchos países con procesos paralelos de democratización y de reforma económica, por lo que algunos autores postularon la existencia de estrechos vínculos entre estos diferentes procesos y la existencia de dinámicas de reforzamiento mutuo. Dichos procesos de descentralización implicaron en concreto la transferencia de responsabilidades, recursos y potestades políticas desde el gobierno central hacia los niveles de gobierno inferiores (estados y municipios) y tuvieron lugar tanto en países federales como unitarios. El resultado fue, en términos generales, una ampliación del poder y la autonomía de las unidades de gobierno subnacionales $y$, en muchos casos, la emergencia de las autoridades políticas subnacionales (gobernadores y alcaldes) como nuevos actores de peso en la política nacional.

La importancia de los procesos de descentralización y los efectos por ellos generados en la vida política de la región dieron lugar a un creciente interés desde el ámbito académico por entender las cualidades adquiridas por las políticas descentralizadoras, así como las razones detrás de las mismas. En efecto, desde entonces pudo constatarse un notable crecimiento de la literatura sobre el tema que, en términos generales, parece haber entendido a la descentralización como el resultado del conflicto y las interacciones estratégicas entre actores políticos nacionales y subnacionales. Esta amplia literatura ha intentado explicar los resultados de los procesos de descentralización, apelando a diferentes factores. Entre los estudios sobre el tema se han destacado los análisis que han puesto el énfasis en los intereses territoriales de los actores institucionales que toman la decisión de descentralizar y el tipo de secuencia que sigue el proceso (Falleti, 2010); el efecto de las crisis económicas en los incentivos de los actores nacionales y subnacionales (González, 2008); la influencia de las dinámicas internas de los partidos y el grado de centralización/descentralización de los mismos (Willis; Garman \& Haggard, 1999; Eaton, 2004); los cálculos electorales de los líderes políticos nacionales acerca del rol que sus partidos jugarán a nivel subnacional en el futuro cercano (O’Neill 2005); motivaciones ideológicas y la noción de que existe un vínculo estrecho entre la descentralización y los procesos de liberalización económica (Eaton, 2013); la existencia de poderes especiales que permitieron a los presidentes negociar de manera particular con las autoridades subnacionales (Eaton, 1998; cited by Escobar-Lemmon, 2001); el intento de los políticos nacionales de ganar nuevamente credibilidad (Rodríguez, 1999); el rol de las instituciones financieras internacionales y la adopción de programas de ajuste estructural; la influencia de las carreras políticas en los cálculos de los actores políticos (Montero and Samuels, 2004); presiones desde abajo como resultado de una creciente competencia electoral (Beer, 2003); solo para mencionar algunos ejemplos ${ }^{1}$.

1. Para un análisis más pormenorizado de las diferentes explicaciones acerca de los procesos de descentralización, puede consultarse Escobar-Lemmon (2001), Montero y Samuels (2004), Falleti (2010).

EL PÉNDULO DESCENTRALIZACIÓN-RECENTRALIZACIÓN Y SU APLICACIÓN A LA REFORMA EDUCATIVA EN MÉXICO 
Ya adentrados en el siglo XXI la ola descentralizadora ha perdido vigor y parece haber dado paso, en un movimiento pendular, a intentos por parte de los gobiernos nacionales de recentralizar el poder, generando como consecuencia una limitación en la autonomía de las unidades subnacionales. Dichos intentos recentralizadores han tenido lugar en diferentes países latinoamericanos, pero también en algunos otros de África y, siendo casos paradigmáticos, también en Rusia y China.

Siendo el eje del presente trabajo, resulta sin embargo necesario ensayar una breve conceptualización sobre lo que entendemos por la idea de recentralización. En este caso, se sigue lo planteado por Eaton y Dickovick, quienes definen la recentralización como "... una serie de cambios diseñados para revertir reformas previas que expandieron la autonomía de los gobiernos subnacionales y, por lo tanto, limitaron las prerrogativas del gobierno nacional" (2004, p.94). Para que resulte claro, las políticas de recentralización son puestas en práctica cuando las autoridades nacionales (los presidentes, en la mayoría de los casos) actúan con la intención de reconquistar el poder sobre recursos y prerrogativas en los ámbitos administrativos, fiscales y políticos que hasta el momento se encontraban bajo el control de las autoridades subnacionales como resultado de procesos descentralizadores ocurridos con anterioridad. A partir de la presente definición debe quedar claro que la recentralización no es intrínsecamente equivalente a la centralización per se, dado que en el caso de la primera existe la intención de regresar a un statu-quo que se vio alterado cuando las políticas descentralizadoras fueron puestas en práctica (Eaton and Dickovick, 2004; Dickovick, 2011a). También puede argumentarse que, desde el punto de vista conceptual, y dado que las políticas recentralizadoras suponen una reducción en los niveles de autonomía de las unidades subnacionales, estas tenderán a ser resistidas por las autoridades subnacionales, ya que suponen una amenaza directa a su poder. Por eso, dichos intentos pueden llevar a un conflicto abierto entre niveles de gobierno y/o a procesos de negociación entre las partes como resultado de los cuales los actores subnacionales acepten dichas limitaciones a sus poderes a cambio de beneficios de otro tipo.

Es importante, sin embargo, dejar en claro que, tal como proponen Dickovick y Eaton (2013), los intentos recentralizadores puede adoptar diferentes formas y ser clasificados de acuerdo con su diversa gradación. De hecho, dichos esfuerzos recentralizadores pueden ir desde medidas recentralizadoras explícitas y de carácter formal (que impliquen cambios legales e institucionales en los marcos que regulan las relaciones entre niveles de gobierno) hasta estrategias más sutiles orientadas a cambiar el balance de poder y reforzar la posición del centro sin que se genere un conflicto explícito con las autoridades subnacionales².

2. Entre dichas estrategias, Dickovick y Eaton (2013) identifican cuatro tipos: estrategias de hechura de políticas públicas, estrategias burocráticas, estrategias institucionales y estrategias societales. Estas estrategias son explicadas con mayor detalle más adelante. 
En este último caso, la autonomía subnacional solo se ve afectada de una manera indirecta: si bien no resulta restringida en el plano legal, sí aparece limitada en términos de su ejercicio efectivo como consecuencia de rol más "central" que asume el gobierno nacional. En este sentido, la recentralización debe entenderse tanto como la reversión de un proceso de devolución de poder a las autoridades subnacionales (Dickovick, 2011a), así como la modificación en el balance de poder en favor del centro en un contexto de post-descentralización.

Así como en el caso de las políticas de descentralización, las políticas recentralizadoras pueden afectar los tres aspectos que moldean la relación entre niveles de gobierno: fiscal, administrativo y político (Falleti, 2010).

La mayoría de las experiencias de recentralización fiscal suponen algún tipo de redefinición acerca de cómo los recursos son distribuidos entre el centro y las unidades subnacionales. Las autoridades nacionales pueden tratar de recentralizar recursos, sea recuperando el control sobre fondos previamente bajo la tutela de los gobiernos subnacionales o bien creando nuevas fuentes de ingresos no compartidos con otros niveles de gobierno. Ejemplos de políticas de recentralización fiscal pueden incluir la creación de nuevos impuestos cuyos ingresos por recaudación se encuentran bajo exclusiva potestad de las autoridades nacionales, cambios en el sistema de transferencias con el objetivo de ampliar la porción correspondiente al centro, la transferencia a los niveles subnacionales de gastos antes erogados por el gobierno nacional sin la descentralización concurrente de los recursos requeridos para financiar dichos pagos, una recentralización de impuestos o recursos previamente descentralizados, un incremento en el uso discrecional de recursos por parte del gobierno nacional, entre otros.

En relación con el plano administrativo, como consecuencia de la recentralización, las autoridades nacionales ganan nuevamente control sobre prerrogativas relacionadas con el diseño e implementación de políticas públicas que se encontraban previamente bajo el control de las autoridades subnacionales y, por lo tanto, aumentan su influencia tanto en relación con la provisión de servicios como en términos de la hechura de las políticas. Esfuerzos recentralizadores en este plano pueden incluir la creación de programas nacionales con impacto directo en la provisión de servicios en el territorio, la fijación de estándares comunes que deben ser cumplimentados por las administraciones provinciales en diferentes áreas de política (como educación, seguridad, salud, etc.), la recentralización de la nómina salarial de los trabajadores provinciales, entre otros.

Finalmente, en términos políticos las políticas de recentralización apuntan a de alguna manera a restringir el espacio de maniobra de las autoridades locales (gobernadores, alcaldes) o a producir "...cambios que limiten la autoridad gubernamental de los funcionarios locales que han llegado al poder como resultado de elecciones" (Eaton, en prensa). En este sentido, ejemplos de recentralización política pueden incluir desde medidas drásticas, como la suspensión de las elecciones locales y el nombramiento de las autoridades subnacionales directamente por orden presidencial o la disolución de unidades subnacionales y su inclusión en regiones autónomas bajo 
la autoridad directa del gobierno nacional, hasta medidas más sutiles, como la introducción de cambios en las instituciones electorales para limitar el influjo de las autoridades subnacionales en las elecciones locales o para "vetar" a ciertos candidatos dispuestos a competir en dichas comicios, el nombramiento de comisionados presidenciales con atribuciones sobre aspectos particulares de la política local, la creación de macro-regiones que crean un nuevo nivel de gobierno intermedio entre el presidente y las autoridades provinciales, entre otros.

Luego de esta breve caracterización de lo que aquí se entiende por recentralización, la siguiente sección propone una revisión algo más detallada de los análisis que en tiempos más recientes han comenzado a surgir sobre las políticas de recentralización y sus causas.

\section{UNA BREVE REVISIÓN DE LA LITERATURA Y ALGUNAS HIPÓTESIS QUE EXPLICAN LA RECENTRALIZACIÓN}

Siguiendo una tendencia similar a lo observado hacia finales del siglo pasado con los trabajos sobre descentralización, la literatura sobre recentralización ha experimentado un crecimiento sustantivo durante años recientes como consecuencia de la recurrencia de procesos de este tipo, aun cuando todavía no ha alcanzado la envergadura de la primera. Una serie importante de análisis se ha enfocado en experiencias observadas en América Latina, tratando de entender las razones, dinámicas y estrategias que subyacen a los intentos recentralizadores.

Así, por ejemplo, Dickovick (2011a) compara movimientos descentralizadores y recentralizadores en países latinoamericanos y africanos y concluye que mientras los gobiernos nacionales tienden a descentralizar cuando enfrentan escenarios de declive político, los presidentes son adeptos a recentralizar una vez que han logrado estabilizar la economía luego de períodos de crisis con efectos hiperinflacionarios. En la misma línea, en un artículo que es citado de manera recurrente, Eaton y Dickovick (2004) analizan procesos de recentralización fiscal observados en Argentina y Brasil durante la década de los 90 y y concluyen que las crisis hiperinflacionarias (y particularmente la manera en la que los gobiernos nacionales son capaces de lidiar con ellas) explican la presencia de políticas recentralizadoras ${ }^{3}$. De acuerdo con este análisis, el éxito de las políticas recentralizadoras es potenciado cuando los presidentes cuentan con influencia sobre sus partidos, cuando existen partidos políticos cohesionados y cuando el presidente es capaz de organizar coaliciones que lo apoyen y conformar gabinetes cohesivos.

Intentos recentralizadores han sido también analizados en relación a la región de los Andes (Ecuador, Bolivia y Venezuela), considerando tanto el discurso desplegado por las autoridades

3. Algunas voces, sin embargo, pusieron en duda, particularmente para el caso de Brasil, que la tendencia recentralizadora identificada por Eaton y Dickovik (2004) estaba en realidad teniendo lugar (ver, por ejemplo, Tavares de Almeida 2005). 
nacionales para justificar dichos intentos (Eaton, en prensa) como las razones que explican que en algunos casos las autoridades subnacionales hayan sido capaces de resistir la agenda recentralizadora del presidente (Eaton, 2013). Presidentes como Hugo Chávez en Venezuela, Rafael Correa en Ecuador y Evo Morales en Bolivia han promovido políticas recentralizadoras orientadas a revertir medidas descentralizadoras anteriores e identificadas por dichos líderes como parte de una agenda neoliberal impulsada por administraciones previas con una visión pro-mercado. Más allá de dicho discurso público, dichos presidentes perseguían además otros objetivos derivados de sus cálculos políticos: apuntaban a reducir las perspectivas electorales de los partidos de oposición que, en todos los casos, eran más poderosos a nivel subnacional y controlaban unidades subnacionales que por sus cualidades económicas tienen peso propio en la vida de dichos países. De hecho, tal como argumenta Eaton (en prensa), la mayoría de las medidas recentralizadoras fueron específicamente dirigidas a unidades subnacionales controladas por la oposición. En un giro interesante, mientras que las políticas recentralizadoras resultaron exitosas en los casos de Ecuador y Venezuela no tuvieron los resultados esperados en Bolivia. De acuerdo con el planteo de Eaton (2013), esta diferencia fue el resultado de la manera en la que los gobiernos subnacionales afectados por estas medidas reaccionarias frente a los intentos recentralizadores: mientras que en el caso de Bolivia los gobernadores de la oposición fueron capaces de movilizar a un número significativo de actores sociales tras el discurso de que la recentralización constituía una amenaza a la identidad regional, este no fue el caso ni en Ecuador ni en Venezuela, en donde la oposición se encontraba más dispersa y tenía posturas contrapuestas en torno a los supuestos beneficios de la descentralización.

Además de buscar explicar las razones detrás de los intentos recentralizadores, una línea paralela de trabajo en la literatura sobre el tema ha intentado categorizar el tipo de estrategias utilizadas por las autoridades nacionales para recentralizar o al menos para buscar mover la balanza de poder a su favor. Así por ejemplo, Dickovick (2011b) argumenta que muchas de las políticas de recentralización son implementadas a partir de la creación de nuevas capas en las instituciones existentes (institutional layering), mientras que Dickovick y Eaton (2013) presentan una tipología de estrategias observadas en países latinoamericanos, orientadas a reforzar el rol del centro en el contexto de escenarios de pos-descentralización. En resumidas cuentas, dichos autores identifican cuatro tipos de estrategias. En primer lugar, las estrategias de política pública hacen referencia a la implementación de programas sociales nacionales con el objetivo de evitar a las autoridades subnacionales y establecer un vínculo directo entre el gobierno nacional y los beneficiarios, lo cual a su vez permite a las autoridades nacionales más fácilmente arrogarse el crédito por las acciones. En segundo lugar, las estrategias burocráticas refieren al uso de la burocracia nacional para impulsar objetivos recentralizadores. En tercer lugar, el gobierno nacional adopta estrategias institucionales cuando intenta "usar instituciones nacionales como las cortes electorales, los cuerpos judiciales y las fuerzas armadas para limitar la autonomía de los gobiernos subnacionales" (Dickovick \& Eaton, 2013, p.1454). Finalmente, las estrategias societales se hacen presente cuando los gobiernos nacionales buscan movilizar actores sociales a nivel subnacional en apoyo a medidas recentralizadoras. 
El énfasis puesto en los casos latinoamericanos no debe sin embargo llevarnos a pensar que este tipo de literatura solo se ha enfocado en nuestra región. Por ejemplo, diferentes análisis han intentado explicar la ola de recentralización que ha tenido lugar en Rusia una vez que Putin ascendió al poder (Konitzer \& Wegren, 2006; Ross, 2005; Sharafutdinova, 2009). En este caso, se argumenta, el crecimiento y extensión territorial del partido de Putin ("Rusia Unida") fue central para reducir el gobierno dividido en términos verticales y crear un ambiente propicio para las medidas recentralizadoras impulsadas por las autoridades nacionales en el plano fiscal, administrativo y, especialmente, político. Asimismo, el estudio de dinámicas similares ha dado también lugar a una extendida literatura para el caso de China, en donde diferentes políticas de recentralización fiscal llevadas adelante durante los años 90 revirtieron la tendencia previa hacia un rol más activo de las aldeas y las ciudades en la economía (Oi et. al., 2012; Ahmad et al, 2002). En su momento, la recentralización fiscal fue utilizada por las autoridades nacionales chinas para incrementar el control administrativo y la vigilancia financiera sobre los funcionarios de las aldeas y las ciudades (Oi et al, 2012).

Tomando en cuenta la literatura sobre el tema, diferentes hipótesis pueden ser identificadas para entender las razones que llevan al éxito de las políticas de recentralización. Al menos existen tres que pueden ser enunciadas. La primera sugiere que los gobiernos unificados (tanto en términos verticales como horizontales), así como la disciplina partidaria, determinan la existencia de un campo propicio para el desarrollo de políticas recentralizadoras. Tomando en cuenta el aspecto horizontal, Eaton y Dickovick (2004) argumentan que el control por parte del presidente del órgano legislativo a través de un partido cohesivo facilita el apoyo a una agenda recentralizadora y explica el éxito de medidas tomadas en dicha dirección. En relación con el aspecto vertical, la recentralización es más probable cuando el mismo partido que controla el gobierno nacional tiene bajo su órbita a una mayoría de las unidades subnacionales. Tal como Eaton (en prensa, p.9) pone en palabras bien claras, "alcaldes y gobernadores que comparten la afiliación partidaria con el presidente se encuentran en un dilema: o bien aceptan la política recentralizadora del presidente y pierden prerrogativas de gobierno por el tiempo que dura su mandato, o bien tratan de defender dichas prerrogativas, se arriesgan a su enojo y enfrentan reprimendas en relación con sus carreras políticas". La experiencia reciente de Rusia provee una evidencia clara de esta situación: las políticas recentralizadoras adquirieron centralidad una vez que el partido de Putin incrementó el control ejercido sobre las unidades subnacionales, lo cual permitió alinear a los funcionarios locales con los deseos del presidente.

Una segunda hipótesis se relaciona con el contexto económico y propone que los presidentes son capaces de recentralizar una vez que han estabilizado la economía luego de una crisis económica (hiperinflacionaria). Atribuyéndose el éxito y presentándose como los salvadores económicos, lo cual en general se traduce en un aumento en sus niveles de popularidad, los presidentes logran en estos contextos incrementar sus poderes para imponer una agenda recentralizadora a actores subnacionales que pueden incluso ser identificados por la población como en parte responsables de la crisis ya superada. Tanto Eaton y Dickovick (2004) como Dickovick (2013) han utilizado esta hipótesis para explicar diferentes experiencias de recentralización tanto en América Latina como en África. 
Por último, una tercera hipótesis alega que las autoridades nacionales estarán más dispuestas a recentralizar cuando creen que las perspectivas de su partido a nivel subnacional son peores que aquellas a nivel nacional o cuando intentan castigar a partidos de oposición que se encuentran en control de uno o varios gobiernos subnacionales.

Más allá del diferente énfasis que cada una de las hipótesis explicativas pone en diferentes factores, todas estas explicaciones comparten dos supuestos básicos. El primero se refiere a los actores identificados como iniciadores de los procesos de recentralización. En este sentido, la premisa asumida es que dado que la recentralización beneficiará con más recursos y prerrogativas al nivel nacional, dichos procesos serán impulsados por las autoridades ejecutivas de dicho nivel de gobierno, en particular por los presidentes. El segundo supuesto se refiere a la particular reacción esperada de quienes son identificados como los principales perdedores de dichos procesos, las autoridades subnacionales (alcaldes, gobernadores). En este caso, la premisa es que dichos actores invariablemente, y por definición, se opondrán a dichos procesos recentralizadores.

A las hipótesis anteriores puede agregarse una cuarta que, sin embargo, no se deriva directamente de la literatura revisada. La misma propone que la recentralización puede tener lugar ante un escenario en el que los gobiernos subnacionales resultan incapaces de brindar de manera efectiva los servicios que en el pasado les fueron transferidos y el gobierno central decide asumir, en parte por necesidad, pero también previendo réditos políticos a futuro, dichas responsabilidades.

Un elemento importante ligado a esta hipótesis es que en este caso la segunda premisa de las expuestas anteriormente puede quedar sin efecto. En otras palabras, bajo estos nuevos preceptos es posible que las autoridades subnacionales no solo no se opongan sino que vean con buenos ojos los intentos recentralizadores.

A partir del marco conceptual expuesto hasta aquí, en la segunda parte de este trabajo se analizan una serie de reformas llevadas adelante recientemente en México que expresan un intento por recentralizar aspectos medulares de la política educativa.

\section{MÉXICO RECIENTE: DESCENTRALIZACIÓN Y RECENTRALIZACIÓN EN EL ÁMBITO EDUCATIVO}

\subsection{EL CONTEXTO}

Durante las últimas décadas del siglo XX, un significativo proceso de descentralización tuvo lugar en México, con efectos importantes en los tres aspectos que regulan las interacciones entre niveles de gobierno: administrativo, fiscal y político (Falleti, 2010; Rodríguez, 1999). 
Algunos autores identifican a la reforma constitucional que en los años 80s modificó el artículo 115 de la Constitución y redefinió el rol de los municipios, incrementando la autonomía de los mismos y garantizando que recibirían de parte de los estados suficientes recursos a través de transferencias fiscales institucionalizadas, como el primer paso hacia la descentralización.

Sin embargo, las reformas más importantes tuvieron lugar en los años 90s cuando se llevó adelante la transferencia de servicios y fueron asignadas a los gobiernos estatales nuevas responsabilidades en la provisión de educación y salud (aspecto administrativo) y las autoridades nacionales decidieron descentralizar una porción significativa del gasto a partir de la creación de un nuevo tipo de transferencias federales (las aportaciones -transferencias federales condicionadas- a ser canalizadas a través del recientemente creado Ramo 33), otorgando a las autoridades subnacionales importantes responsabilidades en la ejecución de una considerable porción del gasto social (aspecto fiscal) (Falleti 2010, Rodriguez 1999).

El Gráfico 1 muestra el impacto sustantivo que esta última reforma generó en la evolución de las transferencias federales a las entidades federativas.

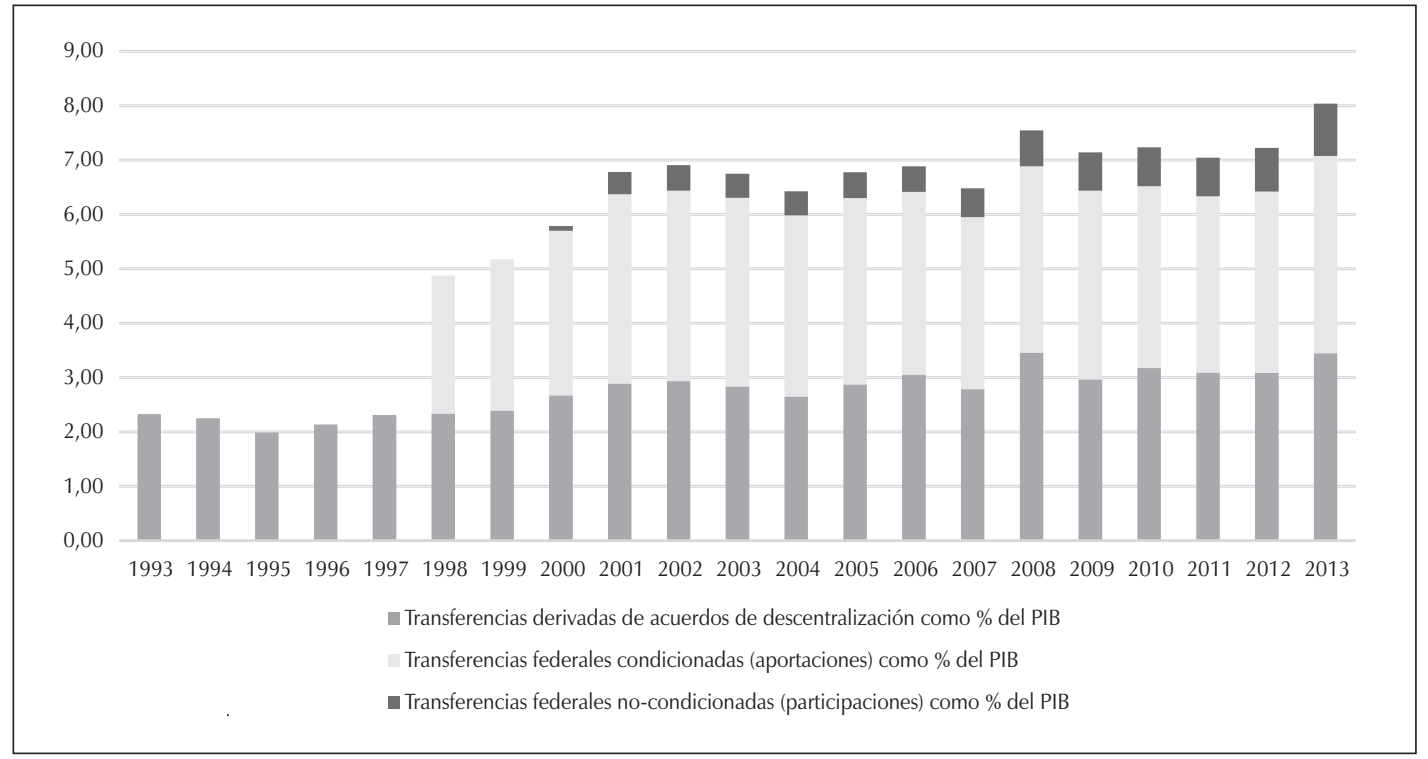

Gráfico 1. Transferencias federales como \% del PIB.

Fuente: Elaboración propia con base en datos de la SHCP.

Tal como puede observarse, a partir de 1998, cuando se crea el Ramo 33, las transferencias recibidas por las entidades federativas se duplicaron, mostrando desde entonces una tendencia ascendente hasta bien entrada la década de los años 2000 cuando se estabilizan. Para 2013, las transferencias federales hacia las entidades federativas por diferentes vías representaban el $8 \%$ del PIB. 
Similares tendencias descentralizadoras pudieron observarse en los 90s en relación con el aspecto político. Por ejemplo, se estableció que autoridades subnacionales que anteriormente eran nombradas por el presidente (en particular el alcalde de la Ciudad de México) pasaran a ser electos por la ciudadanía. Así, en la primera elección para elegir a dicha autoridad resultó victorioso Cuauhtémoc Cárdenas, candidato del opositor PRD, convalidando las autoridades nacionales dichos resultados. Asimismo, se avanzó en la creación, en consonancia con el fortalecimiento a nivel nacional del Instituto Federal Electoral - IFE, de institutos electorales locales encargados de asegurar el desarrollo de elecciones limpias y transparentes. Esto último sin duda contribuyó para que durante la última década del siglo XX creciera de manera significativa el número de candidatos victoriosos de la oposición (principalmente de los dos principales partidos que en ese momento se ubicaban en dicho espacio, PAN y PRD) tanto en contiendas a nivel municipal como estatal.

La combinación de las tendencias observadas en los tres aspectos referidos tuvo como uno de sus efectos más significativos el empoderamiento sustantivo de los gobernadores, quienes pasaron a ser figuras centrales en la vida política nacional. (Hernández Rodríguez, 2008).

La dinámica descentralizadora y el rol cada vez más activo de los gobiernos subnacionales continuó una vez que el PRI perdió las elecciones presidenciales en el año 2000, en parte como resultado del particular escenario que debieron enfrentar los presidentes que siguieron: tanto durante la presidencia de Fox como durante la de Calderón el partido del presidente no logró controlar ni la Cámara de Diputados ni el Senado, teniendo además que lidiar con una mayoría de gobernadores pertenecientes a la oposición.

Durante los años de Fox (2000-2006) los gobiernos subnacionales reafirmaron su autonomía, particularmente a partir del incremento de los recursos bajo su control y transferidos por el gobierno federal (Hernández Rodríguez, 2008). Esto fue el resultado en gran medida del activo rol asumido por la recientemente creada Conferencia Nacional de Gobernadores - CONAGO que, en coordinación con el Congreso Nacional (opositor a Fox), fue capaz de lograr la institucionalización de un fondo especial para obras de infraestructura en los estados (Olmeda, 2013)4. Además, los gobernadores lograron que a partir de entonces el gobierno nacional compartiera con los estados una porción de los codiciados excedentes petroleros que hasta el momento se encontraban bajo el control exclusivo de las autoridades nacionales.

4. La CONAGO fue creada en 2002 por la mayoría de gobernadores de la oposición. Rápidamente, sin embargo, todos los gobernadores -incluyendo aquellos en el partido del presidente- se unieron a la organización, que asumió una postura confrontativa respecto del presidente y tomó el reclamo de una mayor cantidad de recursos para los gobiernos subnacionales como su principal bandera (para análisis más detallados ver Flamand, 2008 y Olmeda, 2013).

EL PÉNDULO DESCENTRALIZACIÓN-RECENTRALIZACIÓN Y SU APLICACIÓN A LA REFORMA EDUCATIVA EN MÉXICO 
Todo esto tuvo lugar en el contexto de crecientes disputas entre el presidente Fox, cada vez más debilitado, y el Congreso. Muchas de las mismas derivaron en un conflicto abierto y consecuentes rechazos a las iniciativas legislativas presidenciales ${ }^{5}$.

Fox solo pudo hacer frente de manera parcial a esta dinámica descentralizadora adoptando lo que, como ya se ha señalado, Dickovick y Eaton (2013) conceptualizan como estrategias recentralizadoras de políticas públicas y burocráticas. En el caso de las primeras, esto se materializó a través del despliegue de un ambicioso programa social de carácter nacional (programa Oportunidades, un rediseño del programa Progresa desarrollado durante la presidencia de Zedillo), y la implementación del programa Escuelas de Calidad, que canalizaba una importante cantidad de recursos a un número de escuelas seleccionadas alrededor del país (la mayoría en zonas pobres) con el objetivo de mejorar la calidad de la educación.

En relación con Oportunidades, Fox debió resistir diferentes embates de las autoridades subnacionales que en diferentes momentos clamaron por su descentralización. Respecto de las estrategias burocráticas, el ejecutivo nacional apunto a impulsar legislación orientada a incrementar el control de las secretarías del gobierno federal sobre la forma en la que las transferencias condicionadas eran gastadas ${ }^{6}$.

Calderón (2006-2012) continuó usando el mismo tipo de estrategias recentralizadoras, especialmente en los sectores de salud y seguridad. En relación con el primero, un nuevo programa diseñado por el gobierno nacional (Seguro Popular) apuntó a proveer cobertura a la población no asegurada, coordinando esfuerzos y recursos tanto del gobierno nacional como de los gobiernos estatales.

En el área de seguridad, la mayoría de los esfuerzos fueron resultado de la llamada "guerra contra el crimen organizado" que se convirtió en una marca registrada de la administración calderonista. La estrategia incluyó un involucramiento activo de las fuerzas armadas en la prevención del delito y la lucha contra los carteles. Además, el gobierno federal promovió el crecimiento de la policía federal y su presencia a lo largo del territorio, al tiempo que se establecieron estándares comunes a ser cumplimentados por todas las fuerzas de seguridad locales.

Finalmente, al igual que lo acontecido durante el gobierno de Fox, el gobierno federal también intentó incrementar su capacidad para supervisar el gasto a nivel subnacional de las transferencias federales condicionadas. Como resultado, diferentes reformas fueron introducidas a la Ley General de Contabilidad Gubernamental con la intención de forzar a los gobiernos subnacionales a hacer sus gastos más transparentes.

5. El fracaso más resonante fue la imposibilidad de lograr sancionar una reforma impositiva que pretendía incrementar y extender la aplicación del IVA impulsada por Fox (ver Langston, 2010).

6. Un ejemplo de este tipo de iniciativas fue la Ley de Presupuesto y Responsabilidad Hacendaria, aprobada por el Congreso en 2006, meses antes del final del gobierno de Fox. 
Sin embargo, fue desde la llegada de Peña Nieto a la Presidencia, en diciembre de 2012, cuando muchos creen ver un cambio real de la tendencia y una profundización del proceso recentralizador, con particulares efectos en el ámbito administrativo y, en particular, en lo referente al sector de política educativa. En una nota de opinión, publicada durante 2013 con motivo del análisis de una serie de iniciativas presidenciales en curso, la reconocida académica María Amparo Casar consideraba que el país era testigo del retorno del centralismo, tanto en doctrina como en práctica, lo cual ponía a su vez en entredicho el propio arreglo federal tan vivo durante años recientes ${ }^{7}$.

Este tipo de dinámica confirmaría que México no ha sido inmune al tipo de tendencias recentralizadoras observadas en otros países latinoamericanos por la literatura detallada en secciones anteriores. Resulta llamativo, sin embargo, que todavía no existan trabajos académicos que analicen la experiencia recentralizadora en México en tiempos recientes de una manera sistemática y profunda. Con la intención de ilustrar la manera en la que opera esta dinámica pendular es que en la siguiente sección se analiza de manera más detallada su materialización en el plano del sector educativo.

\section{EL PÉNDULO DESCENTRALIZACIÓN-RECENTRALIZACIÓN EN ELÁMBITO EDUCATIVO}

La descentralización de la educación constituyó una de las medidas de política más importantes operadas durante el gobierno de Carlos Salinas de Gortari (1988-1994), en el marco de una agenda reformista más amplia que se tradujo en transformaciones sustantivas en el ámbito económico, político, de la política social, y hasta de las vinculaciones internacionales de México.

Dicha descentralización supuso la decisión del gobierno federal de transferir a los estados los servicios educativos hasta entonces en la órbita nacional, asignándoles las potestades para su operación y reservándose las autoridades nacionales responsabilidades normativas y de financiamiento ${ }^{8}$. De acuerdo con diferentes análisis, la transferencia obedeció principalmente a consideraciones del centro antes que a demandas en dicho sentido formuladas por los estados (Ornelas, 1998), argumentándose que la nueva configuración promovería una mayor eficiencia y, en definitiva, serviría para mejorar la calidad del servicio (Pardo, 1999a).

La transferencia se materializó con la firma del Acuerdo Nacional para la Modernización de la Educación Básica y Normal en mayo de 1992, por parte de todos los gobernadores de los

7. Ver el artículo de Casar, M.A. Federalismo de utilería. Recuperado de: http://www.excelsior.com.mx/opinion/mariaamparo-casar/2013/10/09/922546.

8. La transferencia de los servicios educativos fue precedida en décadas anteriores por un proceso de desconcentración administrativa que implicó el establecimientos de 31 delegaciones de la Secretaría de Educación Pública en los estados para asumir las tareas referidas a la operación del sistema (ver Falleti, 2010 y Loyo, 1999)

EL PÉNDULO DESCENTRALIZACIÓN-RECENTRALIZACIÓN Y SU APLICACIÓN A LA REFORMA EDUCATIVA EN MÉXICO 
estados de la república (exceptuando al Distrito Federal, cuya educación básica y normal aún continúa siendo administrada por el gobierno federal), teniendo como testigos de honor a la entonces líder del Sindicato Nacional de Trabajadores de la Educación - SNTE, Elba Esther Gordillo, y al presidente de la república, Carlos Salinas de Gortari. A partir de dicho Acuerdo y los convenios específicos que se firmaron entre las partes, los gobiernos estatales se hicieron cargo de las escuelas y el personal docente que en ella prestaba sus servicios ${ }^{9}$, mientras que el gobierno federal asumió el compromiso de transferir los fondos necesarios para financiar su funcionamiento ${ }^{10}$. Al hacerse cargo de estas nuevas responsabilidades los estados debieron fusionar la porción del sistema educativo transferido con aquellos servicios que ya prestaban en sus territorios. El nuevo balance de responsabilidades y facultades se plasmó asimismo en una nueva Ley General de Educación expedida en 1993, determinando qué potestades serían exclusivas de cada una de las parte y cuáles otras resultarían concurrentes ${ }^{11}$. Mientras que, como ya se ha dicho, la operación se proponía como responsabilidad de los estados, el gobierno federal se reservaba la rectoría general del sistema, asumiendo facultades para la evaluación, la regulación normativa y curricular, así como la implementación de políticas compensatorias que contrarrestasen potenciales disparidades territoriales (Ornelas, 1998). Desde entonces, y con diferente grado de éxito, los presidentes subsiguientes intentaron hacer un ejercicio efectivo de dicho rol rector asignado al gobierno federal. Esto se tradujo en iniciativas que resultaron efectivas en el campo de las políticas compensatorias ${ }^{12}$ y el intento de mejora en los modelos de gestión escolar, con la creación, por ejemplo, del programa de Escuelas de Calidad ${ }^{13}$, pero fracasaron de manera continuada en la intención de evaluar con estándares comunes la preparación y labor de los maestros del sistema ${ }^{14}$.

9. De acuerdo con Ornelas (1998), el gobierno federal transfirió a los estados más de 100,000 edificios escolares e inmuebles, así como 22 millones de bienes muebles, entre vehículos, computadoras, mobiliario escolar, equipo de oficina, etc. Asimismo, se transfirieron 513,000 plazas de maestros y más de 115,000 puestos administrativos. A partir del ciclo escolar 1992-1993 los estados prestaron servicios a cerca de 2’500,000 niños en prescolar, 9 millones en primaria y 75,000 estudiantes de normal (Ornelas, 1998, p.290).

10. Para solventar el financiamiento se creó un nuevo tipo de transferencias federales condicionadas o etiquetadas cuyos recursos deben ser utilizados por los gobiernos estatales exclusivamente para fines educativos. A partir de 1998, dichos recursos comenzaron a transferirse a través del Fondo de Aportaciones para la Educación Básica y Normal FAEB, incluido en el entonces creado Ramo 33 del presupuesto federal.

11. Para un análisis detallado del marco legal, ver Ornelas (1998) y Pardo (1999b).

12. Una revisión de diferentes programas compensatorios puede encontrarse en Pardo (1999b).

13. Este programa se creó al inicio de la presidencia de Fox y tuvo como objetivo transferir recursos desde el gobierno federal directamente a un número seleccionado de escuelas, ubicadas generalmente en zonas de alta marginación, con el objetivo de promover y elevar la calidad de la enseñanza.

14. Quizás el último intento a destacar en dicho sentido tuvo que ver con la firma entre el gobierno federal y el SNTE de la Alianza por la Calidad Educativa, un acuerdo orientado a promover una serie de reformas en la política educativa del país entre las que se encontraban la evaluación externa a los docentes con efectos tanto en las políticas de ingreso como de ascenso. Las evaluaciones implementadas como resultado de la Alianza fueron sin embargo limitadas y ampliamente resistidas por las secciones más combativas del sindicato. 
Por otra parte, los estados asumieron la responsabilidad por la gestión cotidiana, debiendo hacerse cargo la administración tanto de los recursos materiales como humanos que hacen posible la prestación del servicio.

Dicho balance, sin embargo, parece estar modificándose a partir de una serie de movimientos recentralizadores impulsados por el gobierno de Peña Nieto, con importantes y aún inciertas consecuencias en las relaciones de poder entre los niveles de gobierno.

Una de las medidas recentralizadoras más paradigmáticas ha sido resultado directo de la reforma educativa promovida en el marco del Pacto por México ${ }^{15}$. Dicha reforma fue una de las primeras iniciativas de gran envergadura propuestas por el nuevo gobierno e incluyó como una de sus acciones más importantes la creación del Instituto Nacional para la Evaluación de la Educación - INEE. Dicha Institución tiene como una de sus tareas más importantes la evaluación de los maestros a lo largo del país, tanto en lo que respecta a su ingreso a la carrera docente como a lo referido a su promoción y permanencia, siendo que los trabajadores activos que resulten reprobados en una serie de exámenes serán conminados a dejar sus puestos. A través de este movimiento el gobierno federal apunta a recuperar el control sobre la supervisión de las aptitudes y conocimientos con las que deben contar los maestros, siendo que la asignación de plazas parece obedecer hasta ahora a criterios y prácticas que no privilegiaban los méritos (en general producto de acuerdos entre las secciones locales del sindicato y los gobiernos estatales) y la evaluación para la permanencia se había tornado impracticable como resultado de las presiones sindicales.

A esta reforma de más amplio alcance se sumaron medidas recentralizadoras en el ámbito de la administración del sistema educativo. La principal fue la decisión por parte del gobierno federal de recentralizar en su ámbito el control del pago de los salarios de los docentes estatales, función hasta entonces responsabilidad de los gobiernos de los estados. Desde el punto de vista del centro, este movimiento se justificó como una manera de promover una mayor eficiencia económica y la Secretaría de Hacienda resultó el actor que más claramente pugnó por lograr esta redefinición ${ }^{16}$. Junto a esta medida se impulsó la realización de un censo nacional de

15. El Pacto fue resultado de una negociación entre los tres partidos mexicanos más importantes PRI, PAN y PRD en los meses siguientes a la elección de Peña Nieto, en julio de 2012, en el que dichas fuerzas políticas se pusieron de acuerdo acerca de un conjunto de reformas que dichos partidos se comprometían a impulsar y dar sanción en el Congreso una vez que el presidente electo tomara posesión de su cargo. En el Pacto se incluyeron reformas en los ámbitos educativo, fiscal, político, de telecomunicaciones, energético, entre las más importantes. Para mayor detalle ver www.pactopormexico.org.

16. De acuerdo con el secretario de Hacienda, Luis Videgaray, el nuevo esquema permitirá al gobierno nacional tener un mejor control del nombramiento de los maestros, así como sobre los movimientos en la nómina y en los salarios, haciendo el gasto educativo más transparente. Ver Gerardo Hernández (2013, 8 de octubre). El largo brazo de Videgaray. El Zócalo de Saltillo. Recuperado de: http://www.zocalo.com.mx/seccion/opinion-articulo/el-largobrazode-videgaray-1381217575.

EL PÉNDULO DESCENTRALIZACIÓN-RECENTRALIZACIÓN Y SU APLICACIÓN A LA REFORMA EDUCATIVA EN MÉXICO 
todos los trabajadores y estudiantes del sistema educativo en el país con el objetivo de tener información más sistematizada de su tamaño y características.

Más allá de los efectos en términos económicos de estas medidas recentralizadoras, resulta necesario destacar sus importantes implicaciones políticas en el plano de la relación entre niveles de gobierno ${ }^{17}$.

Desde que la provisión de la educación fuera transferida a los estados, la potestad para "contratar y despedir" en el ámbito educativo pasó al ámbito exclusivo de los gobiernos estatales ${ }^{18}$. Dado el significativo peso político del sindicato de los maestros, estas facultades daban a los gobernadores una herramienta importante para negociar con las secciones locales del SNTE beneficios especiales a cambio de apoyo político. La práctica común desde que la educación fuera descentralizada era que las negociaciones entre los representantes de los maestros y las autoridades tuviesen lugar en dos rondas. Primero, el SNTE negociaba con las autoridades nacionales un salario básico común para todos los docentes a lo largo del país. En una segunda ronda, las secciones locales del SNTE negociaban con cada gobernador condiciones de trabajo particulares y beneficios extras para los maestros en cada estado en especial ${ }^{19}$.

La consolidación de esta dinámica de negociación parecía generar intercambios que beneficiaban a las partes involucradas: los afiliados al sindicato lograban importantes concesiones en sus condiciones laborales, mientras que la SNTE ampliaba su influencia en el funcionamiento de la burocracia educativa y la definición de las políticas del sector, entretanto los gobernadores eran capaces de ganar apoyo para reforzar sus maquinarias políticas. El resultado agregado, sin embargo, era un aumento injustificado de la cantidad de plazas y su asignación a partir de criterios de patronazgo antes que de mérito, así como el crecimiento sostenido de la cantidad de maestros en carácter de comisionados, es decir, que no prestan funciones frente a clases.

En paralelo, el uso de criterios formales e informales en la distribución de las transferencias condicionadas para financiar el gasto educativo había comenzado a generar distorsiones que pervertían los objetivos para los que estas habían sido creadas. Desde 2007, la fórmula utilizada para la distribución de las transferencias consideraba, entre otros indicadores, la matrícula escolar, que era medida a partir de un cuestionario completado por los directores de las escuelas. De acuerdo con ciertas críticas, los directores tenían incentivos para "inflar"

17. Ver la nota de opinión del reconocido especialista en temáticas de política educativa Omelas, C. (2014, 5 de marzo). El eslabón débil: los gobernadores. Excelsior. Recuperado de: http://www.excelsior.com.mx/opinion/ carlosornelas/2014/03/05/946982.

18. Sin embargo, como ya se dijo, el pago de los salarios de los trabajadores del sector educativo era financiado a partir de transferencias federales etiquetadas y canalizadas a través de un ramo presupuestal específico (Ramo, 33).

19. Ver Lajous, A. (2013, 28 de febrero). La recentralización de la educación. Nexos. Recuperado de: http://redaccion. nexos.com. $m x / ? p=4626$. 
el número de estudiantes con la intención de justificar plazas y turnos adicionales ${ }^{20}$. Desde el punto de vista informal, se ha documentado que los gobiernos estatales destinaban recursos a actividades que no cumplían con criterios de legalidad (como pagar salarios a maestros con más de una plaza) y que el gobierno federal transfería más recursos a estados en los que se contaba mayor cantidad de movilizaciones de maestros ${ }^{21}$.

Las reformas recentralizadoras ahora implementadas, sin embargo, generarán una redefinición de estas dinámicas: dado que las reformas reforzarán el rol del nivel central, las autoridades nacionales aumentarán su influencia en detrimento de los gobernadores, asumiendo una posición medular en las negociaciones y adquiriendo capacidad para promover una política de beneficios dirigidos a favorecer a determinadas unidades subnacionales.

A pesar de lo anterior, y de que la propuesta de recentralización del pago a los maestros le quita a los gobiernos subnacionales importantes responsabilidades administrativas, la misma ha sido apoyada por varios gobiernos estatales que, en muchos casos, han terminado siendo rehenes de las secciones locales del sindicato. En palabas de Gabino Cué, gobernador de Oaxaca, la centralización del pago de la nómina "reducirá la presión" ejercida sobre las autoridades subnacionales ${ }^{22}$.

\section{CONCLUSIONES}

El análisis anterior parece confirmar que con la llegada de Peña Nieto a la Presidencia el ámbito de la educación ha comenzado a experimentar un proceso de recentralización que estaría trastocando las relaciones entre niveles de gobierno y la dinámica política que ha caracterizado a dicha área desde la descentralización experimentada en la década del 90 del siglo pasado.

Dicha recentralización aparenta estar motivada principalmente por consideraciones de eficacia y eficiencia en la gestión del sistema, lo cual explica el papel central asumido por la Secretaría de Hacienda en el impulso a algunas de las medidas referidas.

Retomando las hipótesis derivadas de la reciente literatura sobre recentralización, es posible realizar una rápida revisión para valorar si alguna de ellas permite explicar el tipo de dinámicas observadas en México. A primera vista, algunas de las hipótesis pueden ser rápidamente

20. Fernández, M.A. (2013, 25 de octubre). Claroscuros de la centralización de la nómina magisterial. México Evalúa.

21. Fernández, M.A. (2012). From the streets to the classrooms: the politics of education spending in Mexico (PhD Dissertation). Duke University.

22. Briseño, P. \& Aguilar, R. (2013, 11 de septiembre). Gobernadores apoyan centralizar los pagos. Excélsior. Ramos, J. \& Robles de la Rosa, L. (2013, 2 de octubre). Recentralizan nómina de SEP; proponen dar medicinas "en especie" a estados. Excélsior.

EL PÉNDULO DESCENTRALIZACIÓN-RECENTRALIZACIÓN Y SU APLICACIÓN A LA REFORMA EDUCATIVA EN MÉXICO 
descartadas. Así, por ejemplo, no es posible argumentar que la recentralización haya coincidido con la salida de una crisis económica que ubicó en una posición fortalecida al presidente. Si bien es cierto que México experimentó una importante contracción en su economía en el período 2008-2010, dicha crisis no compartió el tipo de cualidades observadas en los casos analizados por Eaton y Dickovick (2004) y Dickovick (2011a). En otras palabras, si bien se constató una brusca caída en la actividad, otras variables macroeconómicas (especialmente la inflación) permanecieron relativamente estables. Asimismo, la recuperación económica comenzó años antes de que Peña Nieto asumiera la Presidencia y ciertamente el actual presidente no puede reclamar ningún crédito por dicha evolución favorable (de hecho, durante el primer año de Peña Nieto en la Presidencia el crecimiento del PIB fue menor que el experimentado durante los últimos años de Calderón).

De la misma manera, es posible desestimar la idea de que los movimientos recentralizadores hayan apuntado a castigar de manera estratégica a los gobernadores de la oposición. Efectivamente, las medidas referidas han afectado a las unidades subnacionales como un todo antes que a algunas de ellas en lo particular, al tiempo que el presidente no ha mostrado una actitud confrontativa en relación con los gobernadores de la oposición. Por el contrario, Peña Nieto ha fortalecido su relación con gobernadores prominentes como, por ejemplo, Rafael Moreno Valle, de Puebla (perteneciente al PAN), Miguel Mancera, de la Ciudad de México (perteneciente al PRD), y Manuel Velasco, de Chiapas (perteneciente al Partido Verde). De hecho, después de varios años de demandarlo, la ciudad de México ha conseguido que se le concediera un fondo especial (Fondo de Capitalidad) para compensar los gastos generados por ser la capital del país y que en ella residan los poderes federales y fuese incluida en el Fondo para Infraestructura Social, del que no participaba.

Las dos últimas hipótesis parecen, por el contrario, proveer mejores elementos explicativos. En verdad, el hecho de que el partido del presidente (el PRI) controla una mayoría de las gubernaturas, podría servir para entender por qué los gobernadores no solo desistieron de oponerse a las medidas recentralizadoras sino que actuaron rápidamente cuando las legislaturas locales debieron aprobar la reforma educativa para que esta tuviera efectos constitucionales. Como ya se ha argumentado, los gobernadores oficialistas parece priorizar una buena relación con el ejecutivo federal como condición para potenciar las perspectivas electorales de su partido y, como resultado, hacer avanzar sus carreras políticas en el futuro. Por el otro lado, una resistencia parcial a las reformas parece haber provenido de estados (como Oaxaca y Guerrero) que no solo son gobernados por la oposición sino en los que además se constataron importantes movilizaciones sociales en contra de las reformas motorizadas por las combativas secciones locales del sindicato.

Sin embargo, la hipótesis que mayor fuerza explicativa parece tener es la que pregona que la recentralización obedece a la necesidad del gobierno federal de intervenir a partir de una deficiencia en los servicios prestados. Tal como se adelantó, desde la descentralización se han profundizado en los estados prácticas de reclutamiento del personal docente que no privilegian 
la calidad y el mérito sino que obedecen a acuerdos poco claros entre las secciones locales del sindicato y las autoridades de los gobiernos estatales. Esto no solo ha llevado a un crecimiento injustificado de los privilegios e influencia del sindicato docente sino también al estancamiento de la calidad de la educación que se brinda en las escuelas mexicanas, tal como evaluaciones nacionales como ENLACE o internacionales como PISA parecen atestiguar.

El carácter aún reciente de las reformas aquí analizadas no permite todavía vislumbrar cuáles serán los efectos a largo plazo de estas redefiniciones. Los próximos años permitirán confirmar si la recentralización impacta de manera significativa o no en las dinámicas políticas que hasta hoy día atraviesan la relación entre los niveles de gobierno en México.

\section{REFERENCIAS}

- $\quad$ Ahmad, E.; Keping, L.; Richardson, T. and Singh, R. (2002). Recentralization in China? IMF Working Paper WP/02/168. Washington, D.C.: International Monetary Fund.

- Beer, C. (2003). Electoral competition and institutional change in Mexico. NotreDame, Ind.: Notre Dame University Press.

- Dickovick, T. (2011a). Decentralization and recentralization in the developing world. Comparative Studies from Africa and Latin America. Pennsylvania: The Pennsylvania State University Press, University Park.

- Dickovick, T. (2011b). Recentralization in Latin America: Institutional Layering and Presidential Leverage. Paper delivered at the 2011 Annual Meeting of theAmerican Political Science Association.

- Dickovick, T. and Eaton, K. (2013). Latin America's resurgent centre: nationalgovernment strategies after decentralization. The Journal of Development Studies, 49(11), 14531466.

- $\quad$ Eaton, K. (1998). Political Obstacles to Decentralization in Argentina and the Philippines. Paper presented at the Annual Meeting of the American Political Science Association, Boston, MA, 3-6 september.

- $\quad$ Eaton, K. (2004). The link between political and fiscal decentralization in South America. In Montero, A. \& Samuels, D. (eds.). Decentralization and Democracy in Latin America. Notre Dame, Ind.: Notre Dame University Press.

- $\quad$ Eaton, K. (2013). The centralism of 'Twenty-First-Century Socialism':Recentralising Politics in Venezuela, Ecuador and Bolivia. Journal of Latin American Studies, 45(3), 421- 450. 
- Eaton, K. (forthcoming). Recentralization and the Left Turn in Latin America:Diverging Outcomes in Bolivia, Ecuador, and Venezuela. Comparative Political Studies.

- $\quad$ Eaton, K. \& Dickovick, T. (2004). The politics of re-centralization in Argentina and Brazil. Latin American Research Review, 39(1), 90-122

- Escobar-Lemmon, M. (2001). Fiscal decentralization and federalism in LatinAmerica. Publius: The Journal of Federalism, 31(4), 23-41.

- Falleti, T. (2010). Decentralization and subnational politics in Latin America. New York: Cambridge University Press.

- Fernández, M. A. (2012). From the streets to the classrooms: the politicsof education spending in Mexico (PhD Dissertation). Duke University.

- $\quad$ Flamand, L. (2008). The new role of subnational governments in the federal policyprocess: the case of democratic Mexico. In O'Donnell, G.; Tulchin, J.; Varas, A. \& Stubits, A. (eds.). New Voices in the Study of Democracy in Latin America. Washington D.C.: Woodrow Wilson International Center for Scholars.

- González, L. (2008). Political power, fiscal crises, and decentralization in Latin America: Federal countries in comparative perspective (and some contrasts withunitary cases). Publius: The Journal of Federalism, 30(2), 211-247.

- Hernández Rodríguez, R. (2008). El centro dividido. La nueva autonomía de los gobernadores. México D.F.: El Colegio de México.

- $\quad$ Konitzer, A. \& Wegren, S. (2006). Federalism and Political Recentralization in the Russian Federation: United Russia as the Party of Power. Publius: The Journal of Federalism, 36(4), 503-522.

- Langston, J. (2010). Governors and "Their" Deputies: New Legislative Principals in Mexico. Legislative Studies Quarterly, 35(2), 234-258.

- Loyo, E. (1999). El largo camino de la descentralización educativa 1920-1992. En Pardo, M. del C. (coord.). Federalización e Innovación Educativa en México. México D.F.: El Colegio de México.

- Merino, M. (2003). La transición votada. Ciudad de México: Fondo de Cultura Económica.

- Montero, A. \& Samuels, D. (eds.) (2004). Decentralization and Democracy in Latin America. Notre Dame, Ind.: Notre Dame University Press. 
- Oi, J.; Singer Babiarz, K.; Zhang, L.: Luo, R. \& Rozelle, S. (2012). Shifting Fiscal Control to Limit Cadre Power in China's Townships and Villages. The China Quarterly, 211, 649-675

- Olmeda, J (2013). (Dis)united They Stand? The Politics of Governors' Coalition Building in Argentina, Brazil and Mexico (unpublished PhD Thesis). Northwestern University.

- O'Neill, K. (2005). Decentralizing the State: Elections, Parties, and Local Power in the Andes. New York: Cambridge University Press.

- Ornelas, C. (1998). El ámbito sectorial. La descentralización de la educación en México. El federalismo difícil. En Cabrero Mendoza, E. (coord.). Las políticas descentralizadoras en México (1983-1993). Logros y desencantos. México D.F.: Miguel Ángel Porrúa-CIDE.

- Pardo, M. (1999a). Estudio Preliminar. En Pardo, M. del C. (coord.). Federalización e Innovación Educativa en México. México D.F.: El Colegio de México.

- Pardo, M. (1999b). El órgano central: la tarea normativa y reguladora. En Pardo, M. del C. (coord.). Federalización e Innovación Educativa en México. México D.F.: El Colegio de México.

- Rodríguez, V. (1999). La descentralización en México. De la reforma municipal a Solidaridad y el nuevo federalismo. México D.F.: Fondo de Cultura Económica.

- Ross, C. (2005). Federalism and Electoral Authoritarianism under Putin. In Demokratizatsiya: The Journal of Post-Soviet Democratization, 13(3), 347-371

- Sharafutdinova, G. (2009). Subnational Governance in Russia: How Putin Changed the Contract with His Agents and the Problems It Created for Medvedev. Publius: The Journal of Federalism, 40(4), 672-696.

- Tavares de Almeida, M. (2005). Recentralizando a federação?. Revista de Sociología e Política, 24, 29-40.

- Willis, E.; Garman, C. \& Haggard, S. (1999). The politics of decentralization in Latin America. Latin American Research Review, 34(1), 7-56. 